\section{OC-083 SAME DAY VERSUS SPLIT DOSE BOWEL PREPARATION FOR AFTERNOON COLONOSCOPY: IMPACT ON MUCOSAL VISIBILITY AND PATIENT QUALITY OF LIFE}

doi:10.1136/gut.2011.239301.83

G Longcroft-Wheaton," P Bhandari Gastroenterology, Portsmouth Hospitals NHS Trust, Portsmouth, UK

Introduction Conventional bowel preparation for afternoon colonoscopy involves the use of an oral agent the day prior to the procedure, with further preparation on the morning of the procedure. We hypothesise that for afternoon lists same day bowel prep would be more effective, avoiding a wasted day taking bowel prep and could lead to a cleaner bowel.

Methods Using nationally accepted criteria we compared the recorded quality of bowel preparation. All procedures were performed by the same endoscopist on an afternoon list. Group A consisted of patients prepared with three sachets of picolax, given at 12:00 and 17:00 the day before, and at 8:00 on the morning of the procedure. Group B consisted of patients given two sachets of picolax on the morning of the procedure at 07:00 and 10:00. A Fisher's exact test was performed to test for a significant difference in mucosal visibility between the regimes. Polyp detection, sedation levels and patient comfort was compared between the groups. Patients completed a quality of life (QOL) questionnaire investigating complications, including faecal incontinence, interruption to sleep, headaches, vomiting, abdominal pain, impact on work and social activities and preference of regime.

Results There were 214 patients, 82 in group A and 132 in group B. $70 \%$ were male, median age 65 (range $60-71$ ). $66 \%$ had polyps. There was no difference in sedation or discomfort between the groups. Same day preparation produced better mucosal visibility than the split dose regimen $(p=0.0046)$. See table 1 . Polyp detection in the same day regimen was numerically better ( $71 \%$ vs $62 \%$ ) but the study was not powered to show statistical difference $(\mathrm{p}=0.2)$.
Table 1 OC-083 Quality of mucosal cleansing by bowel preparation regimen

\begin{tabular}{lccc}
\hline & Group A & Group B & P value \\
\hline Excellent & $49.5 \%$ & $46.9 \%$ & \\
Average & $(39.6-59.4)$ & $(38.7-55.5)$ & \\
& $40.0 \%$ & $51.5 \%$ & \\
Poor & $(30.7-50.1)$ & $(43.1-59.9)$ & \\
& $10.5 \%$ & $1.5 \%$ & 0.0046 \\
\hline
\end{tabular}

QOL questionnaires were completed by 92 patients. There was less faecal incontinence, interruption to sleep, headaches, vomiting or abdominal pain with same day prep $(\mathrm{P}=$ 0.0001). Patients in the same day regimen reported less impact on ADLs compared to split dose prep. For the whole cohort patients favoured choice for preparation was for the same day regimen $(p=0.037)$. Amongst the split dose patients $40 \%$ favoured split dose, $48 \%$ same day prep with $12 \%$ unsure ( $\mathrm{P}$ $=0.57)$. Amongst the same day prep patients $14 \%$ favoured split dose, $83 \%$ favoured same day prep with $3 \%$ unsure ( $p$ $=0.0001)$. The difference between the groups was significant $(\mathrm{P}=0.004)$.

Conclusion We found that afternoon colonoscopy with same day bowel preparation is feasible, safe and more effective than the conventional split dose regime. Patients preferred the same day regimen and it has less impact on QOL with less adverse effects. We believe same day bowel prep should become a new standard for afternoon colonoscopy.

Competing interests None.

Keywords bowel preparation, mucosal visibility, quality of life. 